Honam Mathematical J. 36 (2014), No. 3, pp. 689-694

http://dx.doi.org/10.5831/HMJ.2014.36.3.689

\title{
ON THE SUM OF CERTAIN MULTIPLICATIVE FUNCTIONS
}

\author{
InsuK $\mathrm{KIM}^{\dagger, *}$ AND Sungtae Jun
}

\begin{abstract}
We find an asymptotic formula of the sum of multiplicative functions with Dirichlet inversion formula.
\end{abstract}

\section{Introduction}

In studying prime number theorem, several functions, such as Chebyshev function, Mangoldt function and divisor function and etc. are formulated as tools for the investigation of the distribution of primes. Especially, the properties of Mangoldt function and zeta function give a lot of informations of prime numbers. In investigating the distribution of primes, Dirichlet inversion formula has been used to get an asymptotic formula for the sum of Mangoldt function. Thus it is natural to study the sum of a certain mutiplicative function with the inversion formula.

For the other multiplicative functions which we will mention in the lemmas, the general zeta functions can be defined and they utilized in many other problems. However, the similar results can be obtained with exactly the same manner as in the method we do in this paper, except divisor problems.

\section{Multiplicative Functions}

A function $\sigma(n)$ defined on integers is called mutiplicative if $f(m n)=$ $f(n) f(n)$ when $m$ and $n$ are relative prime. Since there are many applications of the generalized zeta functions, it is worthwhile to study the

Received August 18, 2014. Accepted August 28, 2014.

2010 Mathematics Subject Classification. 11R11.

Key words and phrases. zeta function, Dirichlet inversion formula, Mangoldt function.

${ }^{\dagger}$ This paper was supported by Wonkwang University in 2013.

${ }^{*}$ Corresponding author 
properties of some multiplicative functions. For the main theorem, we need the following notations.

For functions $f$ and $g$ with $g \geq 0$, we write that $f(x)=O(g(x))$ or $f(x) \ll g(x)$ when there is a constant $C>0$ such that $|f(x)| \leq C g(x)$. Here $f(x)$ is a complex function of the real variable $x$.

Lemma 2.1. Let $\mu(n)$ be a Möbius function. That is,

$$
\mu(n)= \begin{cases}1 & \text { if } n=1, \\ (-1)^{r} & \text { if } n \text { is a product of } r \text { distinct primes, } \\ 0 & \text { if } n \text { is divisible by the square of a prime, }\end{cases}
$$

and $\zeta(s)=\sum_{n=1}^{\infty} n^{-s}$. Then

$$
\sum_{n=1}^{\infty} \mu(n) n^{-s}=\frac{1}{\zeta(s)} .
$$

Proof. See (1.84) in [2].

Lemma 2.2. Let $\varphi$ be an Euler function defined by

$$
\varphi(n)=\sum_{d \mid n} \frac{n}{d} \mu(d) .
$$

Then

$$
\sum_{n=1}^{\infty} \varphi(n) n^{-s}=\frac{\zeta(s-1)}{\zeta(s)} .
$$

Proof. See (1.109) in [2].

\section{Lemma 2.3.}

$$
\sigma_{a}(n)=\sum_{d \mid n} d^{a} .
$$

Then we have

$$
\sum_{n=1}^{\infty} \sigma_{a}(n) n^{-s}=\zeta(s) \zeta(s-a) .
$$

Remark. If $a=1, \sigma(n)=\sigma_{1}(n)$ is the sum of the divisors of $n$.

Proof. See (1.110) in [2].

Lemma 2.4. The divisors function $d(n)$ defined as,

$$
d(n)=\sum_{d \mid n} 1
$$


which is the number of divisors of $n$. Then

$$
\zeta^{2}(s)=\sum_{n=1}^{\infty} d(n) n^{-s} .
$$

Proof. See (1.104) in [2].

We now find an asymptotic formula of the sum of a multiplicative function with Dirichlet inversion formula, which is the main theorem in this paper. For this, we need the following Perron's formula, which is the special case of the Dirichlet inversion formula. This plays a central role in computing the sum of the above multiplicative functions for $n \leq x$.

Theorem 2.5. (Perron's formula) Suppose that $c>0$. Then

$$
\frac{1}{2 \pi i} \int_{c-i T}^{c+i T} \frac{u^{s}}{s} d s= \begin{cases}1+O\left(u^{c} T^{-1}|\log u|^{-1}\right) & \text { if } u>1, \\ \frac{1}{2}+O\left(c T^{-1}\right) & \text { if } u=1, \\ O\left(u^{c} T^{-1}|\log u|^{-1}\right) & \text { if } 0<u<1 .\end{cases}
$$

Proof. See [1] or [4].

Instead of Theorem 2.5, it is sometimes desirable to have a truncated form of the inversion formula where the integral is over a finite segment whose length may be suitably chosen.

Lemma 2.6. Let $A(s)=\sum_{n=1}^{\infty} a_{n} n^{-s}$ converge absolutely for $\sigma=$ $\operatorname{Re}(s)>1$ and let $\left|a_{n}\right|<C \Phi(n)$, where $C>0$ and for $x \geq x_{0}, \Phi(n)$ is monotonically increasing. Let further

$$
\sum_{n=1}^{\infty}\left|a_{n}\right| n^{-\sigma} \ll(\sigma-1)^{-\alpha}
$$

as $\sigma \rightarrow 1^{+0}$ for some $\alpha>0$. If $w=u+i v$ is arbitrary, $b>0, T>$ $0, u+b>1$, then

$$
\begin{aligned}
\sum_{n \leq x} a_{n} n^{-w} & =(2 \pi i)^{-1} \int_{b-i T}^{b+i T} A(s+w) x^{s} s^{-1} d s+O\left(x^{b} T^{-1}(u+b-1)^{-s}\right) \\
& +O\left(T^{-1} \Phi(2 x) x^{1-u} \log 2 x\right)+O\left(\Phi(2 x) x^{-u}\right)
\end{aligned}
$$

and the estimate is uniform in $x, T, b$ and $u$ provided that $b$ and $u$ are bounded.

Proof. See the appendix in [2]. 
Theorem 2.7. For $t \geq t_{0}>0$ uniformly in $\sigma$,

$$
\zeta(\sigma+i t) \ll \begin{cases}1 & \text { for } \quad \sigma \geq 2, \\ \log t & \text { for } 1 \leq \sigma \leq 2, \\ t^{(1-\sigma) / 2} \log t & \text { for } \quad 0 \leq \sigma \leq 1, \\ t^{(1 / 2-\sigma)} \log t & \text { for } \quad \sigma \leq 0 .\end{cases}
$$

Proof. See Theorem 1.9 in [2].

\section{Main Theorem}

Theorem 3.1. For $T \geq T_{0}>0$ uniformly in $T$, we have (3.1) $\sum_{n \leq x} \varphi(n)=\frac{6}{\pi^{2}} x^{2}+O\left(T^{-1} x^{2} \log x\right)+O\left(\log T(T \log x)^{-1} x^{2}+x(\log T)^{2}\right)$.

Proof. In order to use an inversion formula for Dirichlet series, we consider the following integral,

$$
S(x, T)=\frac{1}{2 \pi i} \int_{c-i T}^{c+i T} \frac{\zeta(s-1) x^{s}}{\zeta(s) s} d s,
$$

where $c=2+(\log x)^{-1}$. Since $\frac{\zeta(s-1)}{\zeta(s)}=\sum_{n=1}^{\infty} \varphi(n) n^{-s}$ converges absolutely for $\sigma=\operatorname{Re}(s)>2$, we assume that $\sigma>2$ from now on.

$$
\begin{aligned}
S(x, T) & =\frac{1}{2 \pi i} \int_{c-i T}^{c+i T} \sum \varphi(n) \frac{(x / n)^{s}}{s} d s \\
& =\sum \varphi(n) \frac{1}{2 \pi i} \int_{c-i T}^{c+i T} \frac{(x / n)^{s}}{s} d s \\
& = \begin{cases}\sum \varphi(n)\left(1+O\left((x / n)^{c}(T \log (x / n))^{-1}\right)\right) & \text { if } x>n, \\
\sum \varphi(n)\left(\frac{1}{2}+O\left((x / n)^{c}(T \log (x / n))^{-1}\right)\right) & \text { if } x=n, \\
\sum \varphi(n) O\left((x / n)^{c}(T \log (x / n))^{-1}\right) & \text { if } 0<x<n,\end{cases}
\end{aligned}
$$

by Theorem 2.5 .

Since we are interested in the case $n \leq x$ only,

$$
S(x, T)=\sum_{n \leq x}^{\prime} \varphi(n)+O\left(\sum_{n=1, n \neq x}^{\infty} \varphi(n)(x / n)^{c}\left(T^{-1}|\log (x / n)|^{-1}\right)\right),
$$

where $\sum^{\prime}$ denotes that the last term in the sum is $\frac{1}{2} \varphi(n)$ if $x$ is an integer. 
Since $c=2+(\log x)^{-1}$, we have $x^{c}=e x^{2}$. Thus

$$
\begin{aligned}
& \sum_{n=1, n \neq x}^{\infty} \varphi(n)(x / n)^{c}\left(T^{-1}|\log (x / n)|^{-1}\right) \\
& \quad \ll x^{2} T^{-1} \sum_{n=1}^{\infty} \varphi(n) n^{-c} \\
& \ll x^{2} T^{-1} \frac{\zeta(c-1)}{\zeta(c)} \\
& \ll x^{2} T^{-1}(\log x), \quad \text { by Theorem } 2.7 .
\end{aligned}
$$

The next step in the proof is to replace the vertical segment of integration $[c-i T, c+i T]$ in $S(x, T)$ by other three sides of the rectangle with vertices $c-i T, c+i T, 1+i T, 1-i T$. Since the integrand in $S(x, T)$ has a pole at $s=2$ with the residue $\frac{x^{2}}{\zeta(2)}$ and it is well known that $\zeta(2)=\frac{\pi^{2}}{6}$

$$
\begin{aligned}
\sum_{n \leq x} \varphi(n)= & \frac{6}{\pi^{2}} x^{2}+O\left(T^{-1} x^{2} \log x\right) \\
& +\frac{1}{2 \pi i}\left(\left(\int_{1+i T}^{1-i T}+\int_{1+i T}^{c+i T}+\int_{c-i T}^{1-i T}\right) \frac{\zeta(s-1) x^{s} d s}{\zeta(s) s}\right) .
\end{aligned}
$$

The integrals in (3.4) will contribute to the error terms in (3.1) and to estimate them we need a suitable bound for $\frac{\zeta(s-1)}{\zeta(s)}$. For $s=\sigma+i T$, if $2<\sigma$, it is easy to see that $\zeta(s)$ has no zeros and is bounded. Thus by Theorem 2.7,

$$
\frac{\zeta(s-1)}{\zeta(s)} \ll \zeta(s-1)=\zeta(\sigma-1+i T) \ll \log T .
$$

Now, we consider

$$
\left(\left(\int_{1+i T}^{1-i T}+\int_{1+i T}^{c+i T}+\int_{c-i T}^{1-i T}\right) \frac{x^{s} d s}{s}\right) .
$$

Since $\frac{1}{|c+i T|} \ll T^{-1}$, we have $\left|\frac{x^{s}}{s}\right| \ll T^{-1} x^{\sigma}$, which implies that

$$
\int_{1 \pm i T}^{c \pm i T} \frac{x^{s}}{s} d s \ll T^{-1} \int_{1}^{c} x^{\sigma} d \sigma=(T \log x)^{-1}\left(x^{c}-x\right) \ll(T \log x)^{-1} x^{c} .
$$


Similary,

$$
\begin{aligned}
\int_{1-i T}^{1+i T} \frac{x^{s}}{s} d s & =\int_{-T}^{T} \frac{x^{1+i t}}{1+i t} i d t \ll \int_{-T}^{T}\left|\frac{x^{1+i t}}{1+i t}\right| d t \\
& =2 x \int_{0}^{T} \frac{1}{\sqrt{1+t^{2}}} d t=2 x \log \left(T+\sqrt{1+T^{2}}\right) \\
& \ll x(\log T) .
\end{aligned}
$$

Since $x^{c}=e x^{2},(3.6)$ are estimated with $\ll(T \log x)^{-1} x^{2}+x(\log T)$. Finally, combining these with (3.5) together, we have that (3.4) « $\log T(T \log x)^{-1} x^{2}+x(\log T)^{2}$.

In the previous section, we mentioned several multiplicative functions. Instead of Euler $\varphi$ function, we are able to find similar results like Theorem 3.1 with the same technique used in the proof. For the case of $\mu(x)$, the result can be found in [2]. For the divisors function, the asymtotic formula of the sum of $d(n)$ is also in [2].

\section{References}

[1] H. Davenport, Multiplicative number theory, 3rd., Springer-Verlag, 2000.

[2] A. Ivic, The Riemann zeta-function, Wiley, 1985.

[3] I. Kim and M. Cho, On the bounds for the sum of the absolute value of Moebius function, Honam Math. J. 36(2) (2014), 467-472.

[4] H. Montgomery, Topics in multiplicative number theory, Springer-Verlag, 1971.

[5] A. Selberg, Contributions to the theory of the Riemann zeta function, Arch. Math. Naturbid. B 48 (1946), 89-155.

[6] A. Selberg, An elementary proof of the prime number theorem, Ann. of Math. 50(2) (1949), 305-313.

Insuk Kim

Department of Mathematical education, Wonkwang University, Iksan, Jeonbuk, 540-749, Korea.

E-mail: iki@wonkwang.ac.kr

Sungtae Jun

Office of general education, Konkuk University,

Chungju, Chungbuk, 380-701, Korea.

E-mail: sjun@kku.ac.kr 